\title{
How to acquire, retain or renew knowledge
}

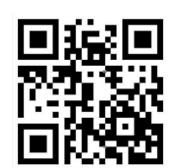

Obstetrics and gynaecology are vast subjects. Each contains 40 elements or so, many of which subdivide into separate topics. Medical students are expected to acquire knowledge of these in 6 weeks, 2 months or 3 months, and will be examined by someone who may have been rereading the information for 25 years. Medical students suffer from a further problem - someone once said, 'What I hear, I forget; what I see, I remember; what I do, I understand.' A student may never see one of the conditions in the exam and may never have been actively involved in the process of choice regarding investigation or management. The result may be that, to the untrained examiner, a bright student may appear ignorant, foolish or careless, and may be inappropriately marked down.

How do students acquire the knowledge required? From textbooks, from university department guidelines, from the internet (if available). Lecturing and bedside teaching are important; these relate to the visual, contextual element of learning. But this learning process is limited by the knowledge of the lecturer or bedside teacher. Knowledge cannot be passed on if the teacher doesn't have it.

The intern, resident or registrar is also a student. Often overworked, they must acquire knowledge at a higher level to face college examiners. Their sources of knowledge are the same as for the medical student.

Beyond medical school or college exams, learning continues. Continuing medical education, or a similar phrase, continuing professional development, is now required by every college and university and by many district local hospitals, where colleagues gather over juice and sandwiches to learn. It continues, or should continue, until the shaking hand lays down the scalpel for the last time.

But what is this knowledge? Clearly, it is the accepted wisdom gathered by the good and the worthy, the many celebrated experts in their field or topic within a subject. That knowledge, however, may not always be completely accurate or correct. It is sometimes difficult to recognise that it is not correct, and difficult to have the courage to accept the enquiring eye of the student (at whatever level) who identifies the inconsistency.

When the emperor walked naked in the parade it was the child and not the cabinet minister who identified the governmental deficit. A senior registrar once criticised as hopelessly inappropriate a student's questioning of maternal weighing in the antenatal clinic in a reasonably nourished population - it neither predicts preeclampsia nor identifies intrauterine growth restriction. The senior registrar marked the student down. The student had gained the gold medal for the year in two of the previous three years. An entire medical school is unlikely to be wrong.

The twin disciplines of obstetrics and gynaecology have been beset with inconsistencies and inaccuracies. It was once believed that patients with regular but heavy menstrual bleeding were anovulatory. This was contained in textbooks; common sense says that that was wrong. It was. Similarly, if a woman is consistently regularly menstrual, tests of ovulation, requested by protocols, were superfluous. Blood sugars were once performed on patients with recurrent miscarriage. Yet how a patient whose difficulty has extended over months or years could be an undiagnosed diabetic and not in a coma was not considered; a brittle established diabetic might, however, suffer with miscarriage. It was once believed that cervical intraepithelial neoplasia should be treated with radiation; the misnomer 'carcinoma in-situ' although not without justification, did not help. Bedrest was recommended for a number of obstetric conditions without ever being evaluated; there was no benefit, and possibly a number of deep-vein thromboses and emboli resulted. The combined oral contraceptive was once given to treat small simple ovarian cysts; that information also appeared in textbooks and guidelines but there is no benefit.

One way to minimise error in knowledge is to put good minds together. This produces college guidelines or the guidelines of accepted bodies and institutions. These guidelines are extremely helpful and excellent for teaching students. They also, however, demonstrate the inconsistencies in obstetrics and gynaecology knowledge is rarely absolute. The American College forbids the use of prostaglandins for the induction of labour of a patient who has had a previous caesarean section, and this is sanctioned by the British College. The American College sanctions the use of misoprostol for induction of labour, but this is forbidden by the British College, fearing overstimulation, unless in a formal research setting. The British College forbids induction of labour where there is intrauterine growth restriction with evidence of fetal compromise. Although this is wise, there has not been such a provision in the American Practice Bulletin, though it seeks to comprehensively advise on the subject.

We rely on the adjudication of governing bodies to help us clarify diseases we see. Yet the International Society for the Study of Vulvovaginal Disease has reclassified vulval disease at least three times in the past 30 years, suggesting that some considerable confusion must have existed at any time during those 30 years. The International Federation of Gynaecologists (FIGO), ${ }^{[1,2]}$ when issuing its classification of abnormal uterine bleeding (the PALMCOEIN system, i.e. polyps, adenomyosis, leiomyoma, malignancy and hyperplasia -coagulopathy, ovulatory disorders, endometrial causes, iatrogenic, not classified), appropriately stated that this classification may be considerably reviewed in ensuing years, perhaps even to the extent of making the current classification unrecognisable. Classification can clarify or cloud, can simplify or confuse.

When we teach and when we learn, guidelines, protocols, research papers and current information in general are a great assistance. They inform our debates and discussions. They assist us in steering away from the turbulence of medicolegal enquiry, rather to tread the path that is accepted and endorsed. But we should not forget that almost nothing is beyond challenge: that the inconsistencies that we cannot see trouble young minds; that it takes courage to accept their criticisms and to realise that we inhibit learning, stifle progress and prevent good logical care of patients by failing to observe what we see; and that when we sit with students or those who come to learn at our scanners or in our operating theatres, incomplete knowledge may hinder and not help.

\section{William Edridge \\ Editor}

1. Munro MG, Critchley HOD, Broder MS, FIGO Working Group on Menstrual Disorders. FIGO classification system (PALM-COEIN) for causes of abnormal uterine bleeding in nongravid women of reproductive age. Int J Gynaecol Obstet 2011;113(1):3-13. [http://dx.doi.org/10.1016/j. ijgo.2010.11.011]

2. Munro MG, Critchley HOD, Fraser IS. The FIGO classification of causes of abnormal uterine bleeding. Int J Gynaecol Obstet 2011;113(1):1-2. [http://dx.doi.org/10.1016/j.ijgo.2011.01.001]

S Afr J Obstet Gynaecol 2015;21(2):26. DOI:10.7196.SAJOG.1045 